\title{
AN OVERVIEW AND CLASSIFICATION OF TOLERANCE COMPENSATION METHODS
}

\author{
Aschenbrenner, Alexander; Schleich, Benjamin; Wartzack, Sandro \\ Friedrich-Alexander-Universität Erlangen-Nürnberg
}

\begin{abstract}
Technological advances as well as novel manufacturing and design paradigms, such as industry 4.0 and digitalization, offer new opportunities for innovative products. However, they also increase the product complexity and cause new challenges in the production process. Therefore, agile production approaches are crucial. Tolerance compensation provides more flexibility in the production process, as demands on dimensional accuracy of the components are reduced. As a result, tolerance compensation also offers the possibility of reducing production costs without compromising product quality. Nevertheless, tolerance compensation is often considered a reactive intervention to reduce the number of out-of-spec parts a posteriori instead of including it in the early stages of Geometrical Variations Management. The contribution tackles this issue by characterizing and categorizing different methods of tolerance compensation as well as providing design guidelines for the application of tolerance compensation methods. This enables design engineers to select a suitable tolerance compensation method for different applications.
\end{abstract}

Keywords: Tolerance Compensation, Robust design, Tolerance representation and management, Uncertainty

\section{Contact:}

Aschenbrenner, Alexander

Friedrich-Alexander-Universität Erlangen-Nürnberg

Engineering Design

Germany

aschenbrenner@mfk.fau.de 


\section{INTRODUCTION}

Novel concepts and ideas in production have emerged inspired by advances in manufacturing technologies, inline measurement systems, and digitalization. Most of them subsume under the term of Industry 4.0 like Mass Customization, Internet of Things, or Digital Twins. These new production concepts either enable or demand for more agile production approaches. What is more, product designs are getting more and more complex. As a result, new challenges for manufacturing and assembly arise. Methods of tolerance compensation may not only provide more flexible production process but can also lead to additional synergy effects if implemented along with one of those new production concepts. For instance, in order to generate a Digital Twin, measurement data is collected during the manufacturing processes of the parts of a product. These data can then be used in real-time for the selection of an optimal mating component (selective assembly) (Lanza, Haefner and Kraemer, 2015)(Wärmefjord et al., 2017) or to plan the adjustment of a compensating element ahead. This allows a quick and flexible reaction to geometric deviations. Hence, a widening of the tolerances of the components becomes possible, which leads to cost savings in the product manufacturing while maintaining product quality.

Nevertheless, tolerance compensation is considered as a reactive intervention to reduce the number of out-of-spec parts a posteriori. This might be one reason why tolerance compensation methods have hardly been discussed in the literature so far - with exception of the selective assembly. Yet, in the context of Industry 4.0, tolerance compensation may become an additional possibility to design robust products that are insensitive to geometric variations. The aim of this contribution is therefore to give a comprehensive overview and classification of different methods of tolerance compensation. Beside the classification, the novelty of this contribution lies in providing qualitative guidelines for the application of the tolerance compensation methods. The contribution is structured as follows: section 2 defines the term tolerance compensation and puts it into the context of the geometrical variations management process and robust design. Thereafter in section 3, different approaches to tolerance compensation are presented, categorized, and requirements for as well as restrictions on their application are outlined. The article closes in section 4 with a short summary and an outlook of future research issues.

\section{TOLERANCE COMPENSATION IN GEOMETRICAL VARIATIONS MANAGEMENT AND ROBUST DESIGN}

In this contribution, tolerance compensation comprises all actions after the manufacturing process or within the assembly process to compensate geometric deviations that would otherwise lead to out-ofspec assemblies or reduce the product quality in an unintended way. However, this excludes design features for tolerance compensation proposed by Litwa et al. (2015) such as sliding flanges or slotted holes. Following Taylor's Principle of go/no-go parts, tolerance compensation includes activities to compensate dimensional deviations violating the upper or the lower limit. A deviation near the golimit (i.e. too much material) can be reworked. On the no-go-limit, impermissible deviations require additional components like shims, springs or washers to compensate for. Other techniques like selective assembly are applicable in both cases.

In general, geometric deviations on real parts are unavoidable due to the imprecision of each manufacturing process and the uncertainty associated with each measurement process. In order to guarantee the assembly, functionality and aesthetics of a product nonetheless, these geometric deviations must be limited. This is the task of the geometrical variation management, which according to Schleich et al. (2018) includes all activities to assure the geometric quality of a product and its subcomponents in all phases of the product life cycle. Thus, tolerance compensation is part of the geometrical variation management. However, the focus within the geometrical variation management is on its main activities, which are tolerance specification, tolerance allocation, and tolerance analysis (Schleich and Wartzack, 2013). Tolerance specification is the definition of the tolerances that are necessary for ensuring the product specification. Afterwards, the values for the selected tolerances are set within the tolerance allocation. Thereafter, the tolerance values can be analyzed with respect to the requirements for assembly, function, aesthetics, or costs. Normally, these three steps are carried out iteratively to achieve a tolerance specification that fulfils all requirements. Yet, tolerance compensation is normally regarded as a last resort to deal with impermissible deviations once the 
components are manufactured. Therefore, the methods of tolerance compensation are hardly taken into consideration during the iterative process of determining an appropriate tolerance specification.

While the geometrical variation management usually deals with geometric deviations originating from the manufacturing and assembly process, according to Howard et al. (2017) robust design goes onestep further and considers variations originating from other (time-dependent) inputs too, like material properties, ambient influences, and operational conditions. The purpose of robust design is therefore the limitation of the performance variation of a product by designing it in a way that it is insensitive to aforementioned input variation. This includes, on the one hand, the investigation of the roots of the variations occurring during the product life cycle and, on the other hand, the analyzation of the effects caused by these variations. The variation management framework (VMF) proposed in (Howard et al., 2017) combines different theories, that are common within the robust design theory, to a holistic approach. The purpose of this approach is to explain and visualize how variations affect a system. Following Suh's Axiomatic Design (Suh, 1989) the product development is segmented into different domains and the product is hierarchically described using Customer Attributes (CA), Functional Requirements (FR), Design Parameters (DP), and Process Variables (PV). To describe the relationship between parameters of the different domains, the VFM employs transfer functions (Oh, 2004). For instance, a transfer function describes how the holding pressure for injection molding (= PV) affects the bore diameter of a component (=DP). An additional transfer function describes how the bore diameter influences the mountability of the assembly (=FR), which may influences a user's perception of the product $(=\mathrm{CA})$. To quantify a customer's perception of the product quality (i.e. the relationship between CAs and FRs), (inverted) Taguchi's Quality Loss Functions (Taguchi, Chowdhury and $\mathrm{Wu}, 2004$ ) are used. By applying these functional relationships, the linkage between the different domains can be represented in quadrants of a coordinate system. Besides this graphical representation, Howard et al. (2017) also provide 7 different levers throughout the quadrants to deal with the quality loss of a product. Especially the levers 2 to 4 are dealt with in greater detail in (Ebro and Howard, 2016) by introducing 15 principles to either reduce the performance variation (lever 2), alter the sensitivity of the transfer function (lever 3) or decrease the variation of design parameters (lever 4). Figure 1 lists all the levers and shows their location within the VMF.

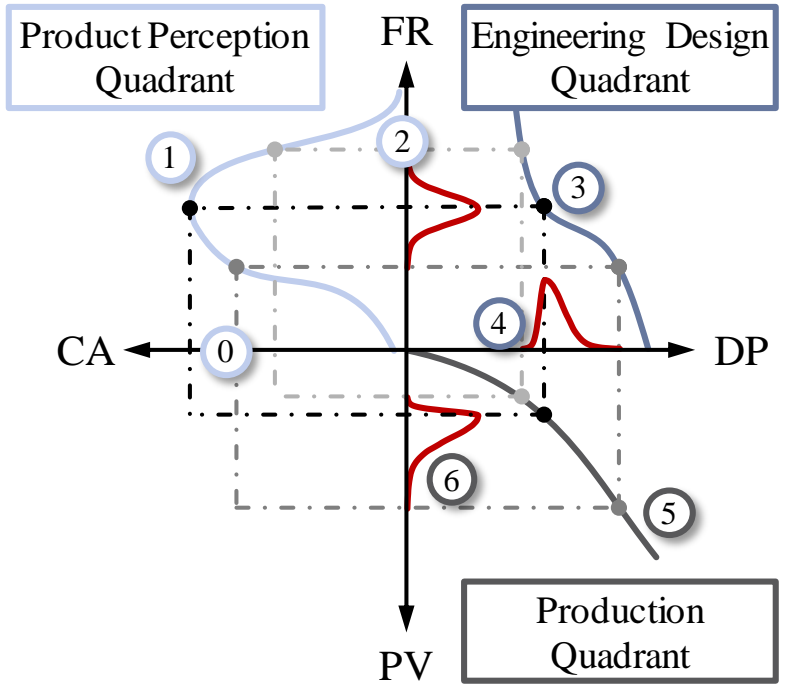

Levers to deal with quality loss
(0) Accept quality loss
(1) Reduce customer's sensitivty to
quality loss
(2) Set target value of the functional
(3) Reduce the gradient of the transfer
(4) Reduce input variation
(5) Reduce effect of the PVs on the DPs
(6) Control and regulate PVs

Figure 1. Variation Management Framework and its levers (based on (Howard et al., 2017))

The methods of tolerance compensations are associated with the levers 2 to 5 . For instance, the use of (post-) assembly adjustments or adjustable elements alters the target value and/or the variation of the functional performance of a product (lever 2). In order to decouple tolerance stack-ups, gaps can be defined and closed by fitting components, like (laminated) shims or supporting rings. Furthermore, flexible elements like springs, tolerance rings or star discs can be employed to compensate gaps. Both options for closing a (foreseen) gap will lead to a less sensitive transfer function (lever 3). Reworking, trimming or selecting matching parts controls the variation of design parameters (lever 4). Of course, selective assembly also reduces how variations in the manufacturing process effect the design parameters (lever 5). The next section describes some of these methods in more detail. 


\section{AN OVERVIEW OF TOLERANCE COMPENSATION METHODS}

Methods of tolerance compensation can be distinguished with regard to the geometry of the assembled parts. There are techniques primarily used for planar components (section 3.1) and other methods that are often used for compact components (section 3.2). Furthermore, there are also methods that are independent of the geometry but more frequently used for compact components like selective assembly. These are also included in section 3.2.

\subsection{Tolerance compensation for planar components}

Methods that are applicable for planar components can compensate, for instance, the deformation of non-rigid sheet metal parts. These methods may as well be beneficial in the challenging field of multimaterial design (aka hybrid design), where a designer has to consider multiple influences, such as the varying thermal expansion and stiffness of the different materials (Rathert, Witzgall and Wartzack, 2018). Examples for such tolerance compensation techniques are the so called tolerance compensation member invented by Schwarzbich (2012) or other systems based on pop rivets (cf. Figure 2). These types of tolerance compensation components are often used for the interior or the exterior of a car or for housings and claddings.

Henning and Moeller (2011) divide the methods for primarily planar components in automatic and manual tolerance compensation. In both cases, the tolerance compensation closes respectively adjusts a gap between two planar parts, as shown in Figure 2. Automatic tolerance compensation has an element with a predetermined breaking point, which triggers as soon as the gap between the two components is closed and, as a result, a certain friction torque is exceeded. Alternatively, there also exist solutions relying on springs, which automatically pull or push a component. In contrast, manual tolerance compensation requires an adjustment process in order to close a gap. Henning and Moeller (2011) further distinguish the tolerance compensation methods with respect to the movement of the compensating element. There is rotatory and translatory tolerance compensation. In case of rotatory tolerance compensation, the compensating element performs a screw movement, whereas in the case of translatory tolerance compensation conical elements are used. Therefore, the compensating element moves translatory. Regardless of the handling (automatic vs. manual) or the direction of movement (rotatory vs. translatory), for most planar tolerance compensation systems a tolerance compensation element is initially fixed to a base plate or part. Then, the component to be aligned must be temporarily fixed within the tolerance zone. Finally, the tolerance compensation elements are tightened and a connection between base part and the now positioned component is established, as shown in Figure 2.

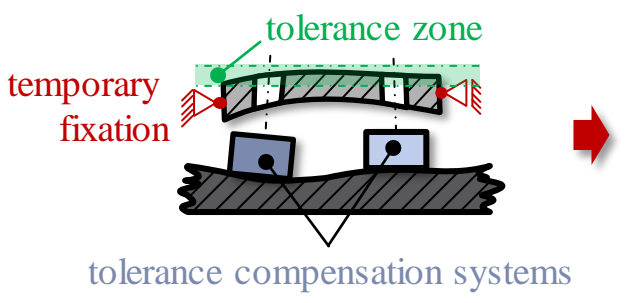

Figure 2. Tolerance compensation for planar components: a) automatic or b) manual tolerance compensation element

Henning and Moeller (2011) also described a couple of areas of application for the planar tolerance compensation methods which are common in the automobile industry, namely compensation of a gap between two parts, blind compensation in case of inaccessible components, compensation of asymmetrical axes and compensation of a misalignment between two adjacent components (flushness or coincidence). Figure 3 depicts these areas of application. A closer look, however, reveals that in both gap and blind compensation, actually only a gap is closed. In fact, the tolerance compensation simply functions as a fixation. Likewise, the cases of symmetry and flushness compensation are very similar too. The difference between the two cases is that in symmetry compensation the tolerance compensation elements act in opposing directions, whereas in flushness compensation they act in the same direction. 


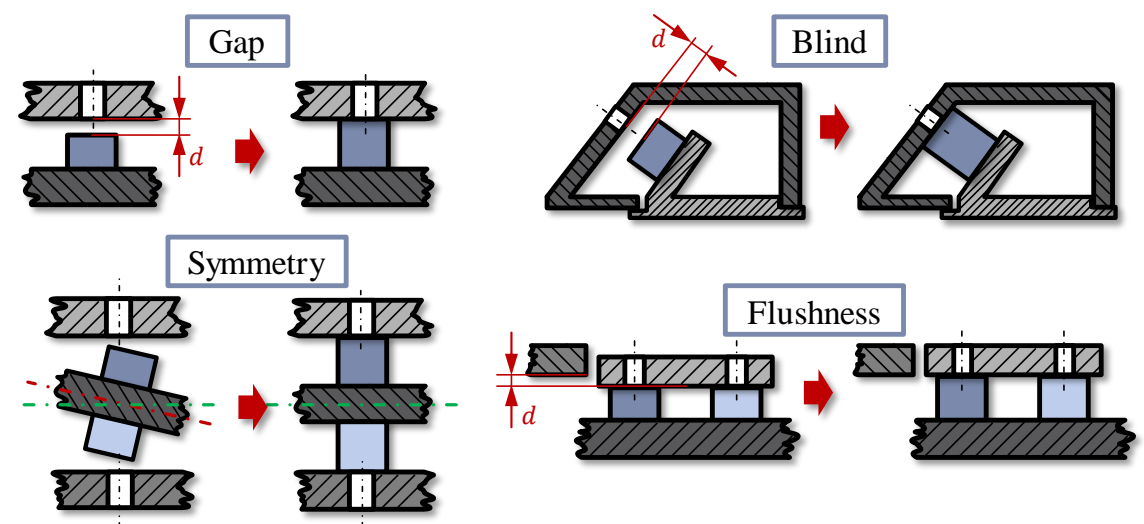

Figure 3. Areas of application of planar tolerance compensation methods according to Henning and Moeller (2011)

In summary, methods for planar tolerance compensation achieve an alignment of a component by altering the distance between this component and a base part in certain support points. However, in the presence of relatively large geometric deviations or deformations due to compliant components, additional support points are necessary or the tolerance zone must be increased. Adding support points, however, increases the manufacturing and assembly costs and, in the case of flexible components, can lead to an unintended behavior of the components due to internal stress. Therefore, it is reasonable to simulate the tolerance compensation in advance in order to optimize the position of the support points. This task is similar to the trimming of locators presented by Lindkvist, Carlson and Söderberg (2005) and therefore will not be discussed further in this article.

\subsection{Tolerance compensation for compact components}

Compact components are normally relatively rigid, like bolts, gears, shafts etc. Similar to tolerance compensation for planar components, tolerance compensation for compact components is mainly used to compensate gaps. For this purpose, additional elements can be used or the production process can be altered. Of course, both categories of tolerance compensation methods may also be applicable for planar components; but are more common for compact components. In the following two subsections, these methods are further classified and are described in greater detail.

\subsubsection{Tolerance compensation by using additional components}

There are two types of elements used for tolerance compensation: rigid elements and adaptive elements. Within a tolerance chain, both types of tolerance compensation elements usually function as a closing part, which compensates dimensional and to some extend geometrical deviations of the other components in an assembly. Rigid elements are further subdivided into elements whose dimensions are continuously variable and can theoretically have any value (e.g. distance washers) and those elements whose dimension are discrete or only available in certain increments (e.g. laminated shims, shim rings or supporting rings (DIN 988)). Figure 4 shows examples of how axial tolerance compensation is obtained for different rigid elements.

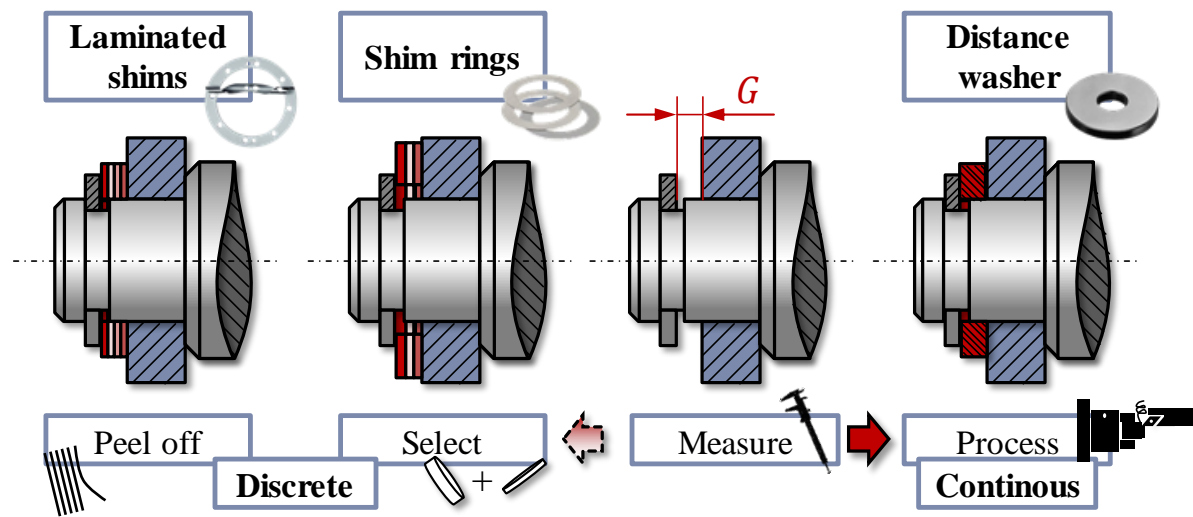

Figure 4. Axial tolerance compensation with rigid elements 
In case of continuous tolerance compensation, the resulting gap is measured and the tolerance compensation element is manufactured according to the measurement. Of course, the dimension of the tolerance compensation element will still vary due to inevitable imprecisions during measuring and manufacturing. Hence, this tolerance compensation option is only beneficial if the expected manufacturing inaccuracy is smaller than the variation of the resulting gap. Furthermore, the compensating element must be manufactured cost-effectively, i.e. the costs for measuring, manufacturing and assembly of the tolerance compensation component must not exceed the additional costs that would be caused by a more accurate manufacturing of the other parts.

Tolerance compensation options that come in discrete dimensions do not require an a priori measuring. Instead, a "trial and error"-like approach can be applied. In case of laminated shims this implies removing as many layers as necessary to leave a gap of the desired dimension. By contrast, when using shim rings, a shim ring of the corresponding thickness is selected or several shim rings are combined in order to obtain a gap that lies within the acceptable limit. Since shim rings come in different width classes, there a various strategies for the combination of the shim rings. For example, similar to a socalled Knapsack Problem (Martello and Toth, 1990), the thickest possible shim ring is selected in each iteration. This approach guarantees a minimum total number shim rings but can lead to a huge variety of width classes. Other strategies may guarantee a maximum number of components within a width class or the use of as less width classes as possible and would therefore be beneficial in the context of the economies of scale. Therefore, when considering the use of classified rigid tolerance compensation components like shim rings, a strategy for combining these parts should be chosen in advance.

Like the continuous tolerance compensation, each element of the discrete compensation element varies in dimension, whereby the variations of the individual components adds up. Moreover, due to the discrete characteristic, a component can only be added or removed in whole. This can increase the risk of an insufficient tolerance compensation (i.e. an out-of-spec gap) compared to continuous tolerance compensation options. On the other hand, discrete tolerance compensation elements are usually very inexpensive. However, the "trial and error"-like approach can be very inefficient, time-consuming and therefore costly. Tolerance simulations can assist a design engineer in estimating these effects beforehand. The other class of tolerance compensation techniques employing additional components comprise adaptive elements. These can be grouped into adjustable and self-adjusting elements. In both cases, some kind of force is necessary to obtain an adaption. For instance, adjustable elements often use threads and screws (sometimes in combination with tapered elements) to create a retaining or clamping force. For axial tolerance compensations, adjustable elements like cone clamping elements, shaft collars, or adjusting rings (DIN 705) can be positioned freely and then are held in position by a clamping force. A taper lock bush fixes a hub to a shaft and compensates radial deviations of the shaft and the bore. For this purpose, screws press the taper lock bush together fixing it to the hub and clamping it onto the shaft. Unfortunately, most of these tolerance compensation techniques can cause unbalanced masses. In addition, high centrifugal acceleration can negate the clamping force. Many adjustable tolerance compensation methods are therefore rather unsuitable for fast rotating applications.

The process of tolerance compensation for adjustable elements is similar to the manual tolerance compensation for planar components (cf. Figure 5): Firstly, a part must be temporarily fixed at the desired positions. Afterwards, the tolerance compensation component is tightened. This approach enables to transfer a serial assembly into a parallel assembly without couplings between the individual components (Söderberg and Lindkvist, 1999). The accuracy of the position of a part respectively the accuracy of the tolerance compensation now mainly depends on the accuracy of the positioning as well as the tightening rather than the dimensional accuracy of the parts.

Besides adjustable elements, there are also self-adjusting elements within the group of adaptive elements. Tolerance compensation is usually achieved by means of elastic deformation of the elements. Examples are spring washers (DIN 42013), star discs, springs, Seeger ${ }^{\circledR}$ beveled rings, or tolerance rings (DIN 42020) for radial tolerance compensation. Figure 5 depicts schematically the application of self-adjusting tolerance compensation for the example of springs and beveled rings.

In both cases, the self-adjusting element is first elastically deformed to enable the assembly of all parts (this is achieved by either pressing the tolerance compensation element together (spring) or pushing it apart (beveled ring)). By releasing the tolerance compensation element, it aligns with its surrounding parts. This creates an equilibrium of forces that holds all parts in place and in some cases allows to preload components (e.g. roller bearings). However, in applications in which the equilibrium of forces cannot be achieved, e.g. due to additional forces, this type of tolerance compensation is not applicable. 
Self-adjusting components reduce the number of parts or rather surfaces involved in a tolerance stack-up, which is often critical within an assembly (Söderberg and Johannesson, 1999). Moreover, self-adjusting components completely close existing gaps. For instance, in case of the beveled ring in Figure 5 the position of the blue part only depends on its own width and the shoulder of the shaft. The beveled ring aligns itself accordingly and thus, there is no gap between the blue part, the shoulder and the beveled ring.

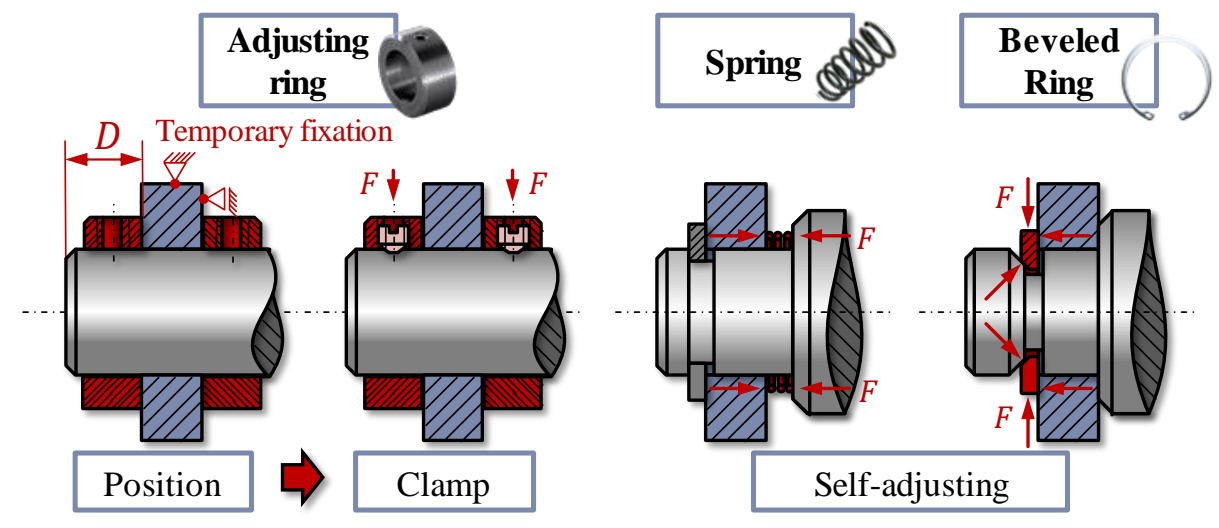

Figure 5. Examples of axial tolerance compensation using adaptive elements

In general, tolerance compensation using additional components implies modified or additional assembly operations. In addition, these tolerance compensation elements normally require extra space or at least a modified design of the surrounding parts (e.g. in case of tolerance rings or beveled rings). All these modifications can increase the production costs. Hence, tolerance compensation using additional components is only reasonable, if these drawbacks are made up for by a higher quality of the product or by reduced tolerance-related cost of the other parts.

\subsubsection{Tolerance compensation by modifying the manufacturing or the assembly process}

Tolerance compensation can also be achieved by modifications within the production process. If a stack-up exceeds its go-limit, the simplest way of tolerance compensation is to rework one or more parts. Obviously, this is only possible if at least one part exists, that will not violate its no-go-limit after remanufacturing. The advantage of reworking is the reduction of the scrap rate without any changes to the actual manufacturing or assembly process. Nevertheless, reworking results in unnecessarily increased production costs and should therefore be the exception rather than the rule. Instead, if components tend to be manufactured near their go-limit, Statistical Process Control and Root Cause Analysis can help to improve the accuracy of manufacturing processes (Wärmefjord et al., 2017).

Selective assembly is another, more powerful way to control the variation of an assembly, especially in the case of quality requirements close to technological limits (Lanza, Haefner and Kraemer, 2015). The intention of selective assembly is to produce high precision assemblies from low precision components (Colledani, Ebrahimi and Tolio, 2014). In fact, the use of selective assembly covers a large variety of applications, like recycled parts (Liu, Liu and Zhu, 2014), high-end electronics (Ebro and Howard, 2016), as well as high precision parts like roller bearings (Aschenbrenner and Wartzack, 2017). For selective assembly it is necessary to classify parts of the same type according to their size and to divide them into several groups before assembly (Mansoor, 1961). These steps are normally performed for two or more types of parts, which are mating during the assembly process. During the assembly process, parts from corresponding groups are matched und thus, variation is cancelled out to some degree. Alternatively, compensating elements are classified and grouped. Then, a measurement is performed during the assembly process and a compensating element of a corresponding group is selected (almost like the previously presented tolerance compensation using continuous components). However, selective assembly also has its disadvantages. For example, selective assembly by definition requires a categorization of the different components. This can result in a time-consuming and costly inspection and control process. In addition, the expenses for separating and storing the classified components may also increase the production costs. Moreover, the replacement of components in case of maintenance may not be possible, because no matching component exists (Ebro and Howard, 2016). Furthermore, the number of surplus parts increases undesirable, if the distribution of the components differ. Luckily, grouping methods like the one proposed by Chan and Linn (1998) have already been developed for this issue. 
Another tolerance compensation option, which modifies the production process, is the optimization of the joining sequence. It is based on the idea that there are sequences for which geometric deviations are (partly) cancelled out (Wärmefjord et al., 2017). Depending on the composition of the components, this implies either a temporal or a spatial change in the installation sequence. A spatial change is of course only possible if the type of the components is identical. Unlike the other tolerance compensation methods, the joining sequence optimization targets the compensation of geometric deviations rather than dimensional deviations. Therefore either an inefficient trial-and-error approach is applied or scan-data in combination with sophisticated tolerance simulation models like skin model shapes (Schleich et al., 2016) are employed to determine an optimal joining sequence. Of course, this implies an accurate (in-situ) measurement of the geometric deviations for the real parts (Wärmefjord et al., 2017).

\section{CONCLUSION AND OUTLOOK}

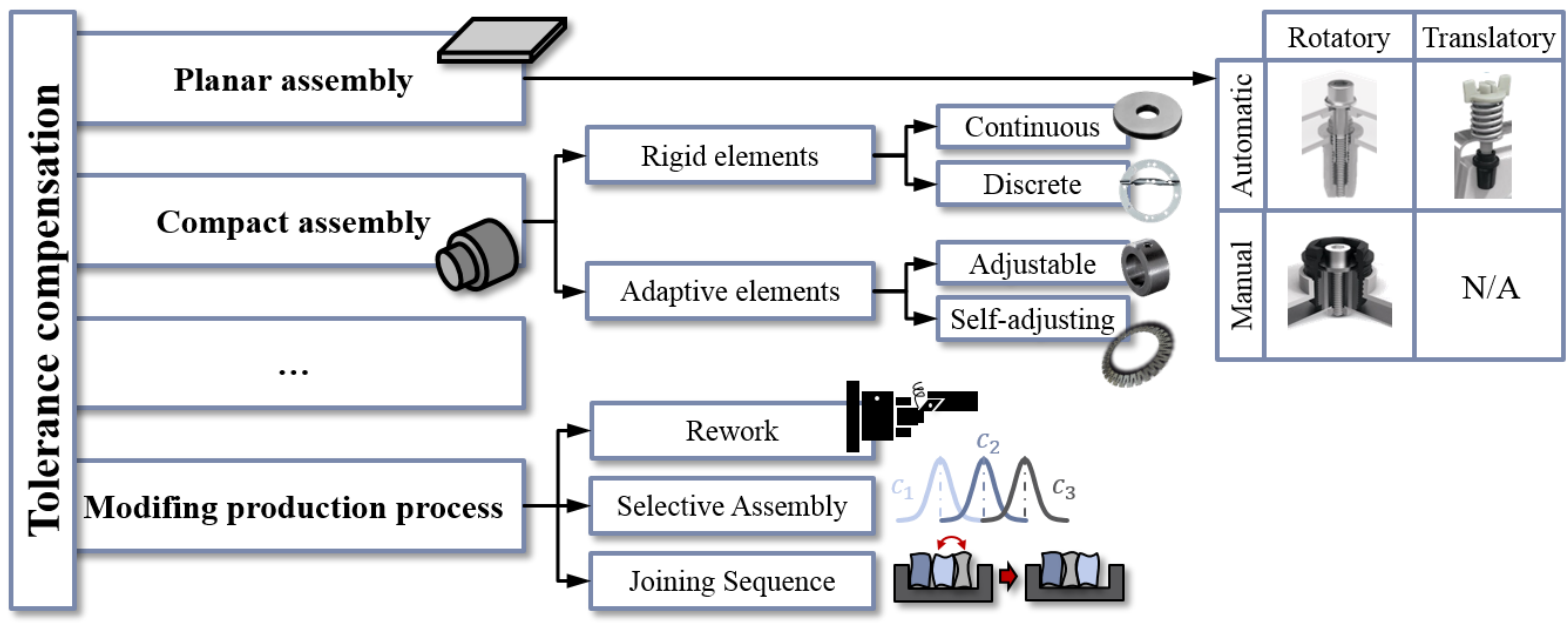

Figure 6. Categorization of tolerance compensation methods

Figure 6 visualizes the classification of the tolerance compensation methods presented in this article. To summarize, most of the tolerance compensation methods compensate dimensional deviations, i.e. they close gaps. There are tolerance compensation methods primarily used for assemblies of planar parts and other methods that are more common for assemblies of compact parts.

Planar tolerance compensation methods are often used to ensure aesthetic requirements by guaranteeing closed gap, symmetry, or flushness of components. For these tasks, normally a base part is necessary. Yet, the tolerance compensation are able to substitute other types of fixations. A design engineer should keep in mind, that using planar tolerance compensation elements in multiple support points can influence the behavior of compliant assemblies in an unintended way, due to internal stress. Tolerance compensation methods for compact assemblies often use additional parts. These elements are further subdivided into rigid and adaptive elements. In both cases, a design engineer should be aware of extra costs associated with design changes (especially for adaptive elements), increased measurement efforts, and modified or additional production steps. Therefore, a design engineer should consider whether the increased quality or reduced tolerance costs of the other components overcome these disadvantages. If employing continuous rigid elements, the variation of the compensating element should be smaller than the cumulative variation of the other components. On the other hand, discrete tolerance compensation elements may not guarantee the closing of a gap, as a compensating component can only be added respectively removed as one. For classified discrete tolerance compensation methods, a strategy for the combination of the compensating components is furthermore required. Adaptive tolerance compensation elements use forces in some way to realize tolerance compensation. On the one hand, these forces must not negatively influence the behavior of the assembly, like unbalanced masses or excessive preloading. On the other hand, the forces must be sufficient during operation to ensure the positioning of the components.

Alternatively to additional components, modifications of the production process are often applied for compact assemblies. Reworking is the easiest way to compensate deviation of components with too much material. However, reworking should be avoided. Instead, a stable manufacturing process should be established using Statistical Process Control and Root Cause Analysis. Selective assembly, on the other 
hand, is a powerful approach to enable high precision assemblies with low precision components. However, it is not applicable for maintenance-intensive products and the extra costs for classifying and combining parts should not exceed the benefits of an increased product quality and/or lower manufacturing costs. Furthermore, a strategy for the classification is required to answer questions like how many classes are needed, how to combine classes, or what to do with surplus parts. Another high potential tolerance compensation method is the optimization of the joining sequence, as geometric rather than dimensional deviations can be compensated. To exploit this potential, however, in-situ measuring along with real-time optimization based on sophisticated tolerance simulation are necessary.

Obviously, the list of the tolerance compensation methods described in this contribution is not complete. For instance, components can be designed in a way that allows an adaption during assembly. There are also production techniques that generate functional surfaces during the assembly process and are therefore insensitive to geometric deviations, like clinching or flanging. These techniques can be regarded as an additional type of tolerance compensation methods (Litwa et al., 2015). What is more, innovative and flexible manufacturing processes like Additive Manufacturing can be used ad-hoc to manufacture compensating elements for complex geometrical variations using scan-data.

In general, product development and production shifts more and more towards real-time simulations and optimization, due to more efficient algorithms, an ever-increasing computing power, as well as a growing quality and quantity of data (Söderberg et al., 2017). Furthermore, highly realistic virtual product models like Skin Model Shapes are gaining increased relevance for the assurance of the product quality along the product life cycle (Schleich et al., 2017). To provide a design engineer not only with qualitative but also quantitative guidelines for the use of tolerance compensation methods, integration of tolerance compensation methods in sophisticated virtual simulation models is needed. This includes a highly realistic sampling of tolerance compensation elements as well as the simulation of the physical behavior of these elements. Moreover, the costs and the qualitative benefits of the individual methods must be evaluated a priori to their use. Therefore, new tolerance cost models and adapted tolerance cost optimization are necessary. For the evaluation of the influence of a tolerance compensation method on product quality, sensitivity analysis can be applied. However, variance-based sensitivity is not suitable for all tolerance compensation methods. For instance, when using selective assembly, sensitivity must be calculated for each group separately; otherwise, statistical problems arise due to correlating groups (Aschenbrenner, Schleich and Wartzack, 2017). Therefore, other, more sophisticated methods of sensitivity analysis may be beneficial like density-based sensitivity analysis (Schleich and Wartzack, 2018) or fuzzy sensitivity algorithm (Oberleiter et al., 2018). These and further topics should be tackled in future researches.

\section{REFERENCES}

Aschenbrenner, A., Schleich, B. and Wartzack, S. (2017), "The influence of sorting on the statistical behavior of functional dimensions", 28th Symposium Design for X (DFX 2017), Bamberg/Germany, 4th-5th October 2017, TuTech Verlag, Hamburg/Germany, pp. 75-86.

Aschenbrenner, A. and Wartzack, S. (2017), "A Method for the Tolerance Analysis of Bearing Seats for Cylindrical Roller Bearings in Respect To Operating Clearance and Fatigue Life", 21st International Conference on Engineering Design (ICED 17), Vancouver/Canada, 21st - 25th August 2017, Design Society, Glasgow, pp. 79-88.

Chan, K.C. and Linn, R.J. (1998), “A grouping method for selective assembly of parts of dissimilar distributions”, Quality Engineering, Vol. 11 No. 2, pp. 221-234. http://dx.doi.org/10.1080/08982119808919233.

Colledani, M., Ebrahimi, D. and Tolio, T. (2014), "Integrated quality and production logistics modelling for the design of selective and adaptive assembly systems", CIRP Annals, Vol. 63 No. 1, pp. 453-456. http://dx.doi.org/10.1016/j.cirp.2014.03.120.

Ebro, M. and Howard, T.J. (2016), "Robust design principles for reducing variation in functional performance", Journal of Engineering Design, Vol. 27 No. 1-3, pp. 75-92. http://dx.doi.org/10.1080/09544828.2015.1103844.

Henning, F. and Moeller, E. (2011), Handbuch Leichtbau, Carl Hanser Verlag GmbH \& Co. KG, Munich/Germany. http://dx.doi.org/10.3139/9783446428911.

Howard, T.J., Eifler, T., Pedersen, S.N., Göhler, S.M., Boorla, S.M. and Christensen, M.E. (2017), “The variation management framework (VMF): A unifying graphical representation of robust design", Quality Engineering, Vol. 29 No. 4, pp. 563-572. http://dx.doi.org/10.1080/08982112.2016.1272121. 
Lanza, G., Haefner, B. and Kraemer, A. (2015), "Optimization of selective assembly and adaptive manufacturing by means of cyber-physical system based matching”, CIRP Annals, Vol. 64 No. 1, pp. 399-402. http://dx.doi.org/10.1016/j.cirp.2015.04.123.

Lindkvist, L., Carlson, J.S. and Söderberg, R. (2005), "Virtual Locator Trimming in Pre-Production: Rigid and Non-Rigid Analysis", ASME International Mechanical Engineering Congress and Exposition (IMECE), Orlando/USA, 5th-11th November 2005, ASME, pp. 561-568. http://dx.doi.org/10.1115/IMECE200581266.

Litwa, F., Gottwald, M., Forstmeier, J. and Vielhaber, M. (2015), "Determination Of Functional Intersections Between Multiple Tolerance-Chains By The Use Of The Assembly-Graph", NAFEMS World Congress 2015, San Diego/USA, 21st-24th June 2015, pp. 189-203.

Liu, M., Liu, C. and Zhu, Q. (2014), “Optional classification for reassembly methods with different precision remanufactured parts", Assembly Automation, Vol. 34 No. 4, pp. 315-322. http://dx.doi.org/10.1108/AA03-2014-023.

Mansoor, E.M. (1961), "Selective assembly - its analysis and applications", International Journal of Production Research, Vol. 1 No. 1, pp. 13-24. http://dx.doi.org/10.1080/00207546108943070.

Martello, S. and Toth, P. (1990), Knapsack Problems: Algorithms and Computer Implementations, John Wiley $\&$ Sons, Inc., New York/USA.

Oberleiter, T., Heling, B., Schleich, B., Willner, K. and Wartzack, S. (2018), "Fuzzy Sensitivity Analysis in the Context of Dimensional Management", ASCE-ASME Journal of Risk and Uncertainty in Engineering Systems, Part B: Mechanical Engineering, Vol. 5 No. 1, pp. 011008-011008-7. http://dx.doi.org/10.1115/1.4040919.

Oh, H.L. (2004), "Unifying axiomatic design and robust design through the transfer function", The Third International Conference on Axiomatic Design (ICAD2004), Seoul/South Korea, 21st-24th June 2005, pp. $1-4$.

Rathert, T., Witzgall, C. and Wartzack, S. (2018), "Modular rapid design of multi-material lightweight truss structures - A novel approach", Symposium Lightweight Design in Product Development, Zurich/Switzerland, 14th-15th June .2018, CMASLab, ETH Zurich, Zurich/Switzerland, pp. 21-23. http://dx.doi.org/10.3929/ethz-b-000283432.

Schleich, B., Anwer, N., Mathieu, L. and Wartzack, S. (2016), "Status and Prospects of Skin Model Shapes for Geometric Variations Management”, Procedia CIRP, Vol. 43, pp. 154-159. http://dx.doi.org/10.1016/j.procir.2016.02.005.

Schleich, B., Anwer, N., Mathieu, L. and Wartzack, S. (2017), "Shaping the digital twin for design and production engineering”, CIRP Annals, Vol. 66 No. 1, pp. 141-144. http://dx.doi.org/10.1016/j.cirp.2017.04.040.

Schleich, B., Wärmefjord, K., Söderberg, R. and Wartzack, S. (2018), "Geometrical Variations Management 4.0: Towards next Generation Geometry Assurance”, Procedia CIRP, Vol. 75, pp. 3-10. http://dx.doi.org/10.1016/j.procir.2018.04.078.

Schleich, B. and Wartzack, S. (2013), “"How to determine the influence of geometric deviations on elastic deformations and the structural performance?"', Proceedings of the Institution of Mechanical Engineers, Part B: Journal of Engineering Manufacture, Vol. 227 No. 5, pp. 754-764. http://dx.doi.org/10.1177/0954405412468994.

Schleich, B. and Wartzack, S. (2018), "An Approach to the Sensitivity Analysis in Variation Simulations considering Form Deviations", Procedia CIRP, Vol. 75, pp. 273-278. http://dx.doi.org/10.1016/j.procir.2018.03.314.

Schwarzbich, J. (2012), US patent US9074614B2: "Tolerance compensation member".

Söderberg, R. and Johannesson, H. (1999), "Tolerance Chain Detection by Geometrical Constraint Based Coupling Analysis", Journal of Engineering Design, Vol. 10 No. 1, pp. 5-24. http://dx.doi.org/10.1080/095448299261399.

Söderberg, R. and Lindkvist, L. (1999), “Computer Aided Assembly Robustness Evaluation”, Journal of Engineering Design, Vol. 10 No. 2, pp. 165-181. http://dx.doi.org/10.1080/095448299261371.

Söderberg, R., Wärmefjord, K., Carlson, J.S. and Lindkvist, L. (2017), "Toward a Digital Twin for real-time geometry assurance in individualized production”, CIRP Annals, Vol. 66 No. 1, pp. 137-140. http://dx.doi.org/10.1016/j.cirp.2017.04.038.

Suh, N.P. (1989), The principles of design, Oxford University Press, New York/USA.

Taguchi, G., Chowdhury, S. and Wu, Y. (2004), Taguchi's Quality Engineering Handbook, John Wiley \& Sons, Inc., Hoboken/USA. http://dx.doi.org/10.1002/9780470258354.

Wärmefjord, K., Söderberg, R., Lindkvist, L., Lindau, B. and Carlson, J. S. (2017) "Inspection Data to Support a Digital Twin for Geometry Assurance”, ASME 2017 International Mechanical Engineering Congress and Exposition, Tampa/USA, 3rd-9th November 2017, ASME, http://dx.doi.org/10.1115/IMECE2017-70398. 